\title{
ASYMPTOTIC ANALYSIS OF A FLUID MODEL MODULATED BY AN M/M/1 QUEUE
}

\author{
CHARLES KNESSL, ${ }^{*}$ University of Illinois at Chicago \\ DIEGO ERNESTO DOMINICI,** State University of New York at New Paltz
}

\begin{abstract}
We analyze asymptotically a differential-difference equation that arises in a Markovmodulated fluid model. We use singular perturbation methods to analyze the problem with appropriate scalings of the two state variables. In particular, the ray method and asymptotic matching are used.
\end{abstract}

Keywords: Fluid queue; asymptotics; M/M/1 queue; ray method

2000 Mathematics Subject Classification: Primary 60K25

Secondary 60J25; 34E10

\section{Introduction}

Fluid models have received much recent attention in the literature. They have been used to model statistical multiplexers in ATM (asynchronous transfer mode) networks [11], [12], [15], packet speech multiplexers [16], buffer storage in manufacturing models [19], buffer memory in store-and-forward systems [5], and high-speed digital communication networks [9]. In these models the queue length is considered a continuous (or 'fluid') process, rather than a discrete random process that measures the number of customers. These models tend to be somewhat easier to analyze, as they allow for less randomness than more traditional queueing models.

The following is a description of a fairly general fluid model, of which many variants and special cases have been considered. Let $X(t)$ denote the amount of fluid at time $t$ in the buffer. Furthermore, let $Z(t)$ be a continuous-time Markov process. The content of the buffer $X(t)$ is regulated (or driven) by $Z(t)$ in such a way that the net input rate into the buffer (i.e. the rate of change of its content) is $\eta[Z(t)]$. The function $\eta(\cdot)$ is called the drift function. When the buffer capacity is infinite, the dynamics of $X(t)$ are given by

$$
\frac{\mathrm{d} X}{\mathrm{~d} t}= \begin{cases}\eta[Z(t)], & X(t)>0 \\ \max \{\eta[Z(t)], 0\}, & X(t)=0 .\end{cases}
$$

The condition at $X(t)=0$ ensures that the process $X(t)$ does not become negative.

In many applications, the process $Z(t)$ evolves as a finite or infinite state birth-death process. The description of the motion of $Z(t)$ is as follows: the process sojourns in a given state $k$ for a random length of time, whose distribution is exponential with parameter $\lambda_{k}+\mu_{k}$. The motion is analogous to that of a random walk, except that transitions occur at random rather than fixed times. The parameters $\lambda_{k}$ and $\mu_{k}$ are respectively called the birth and death rates.

Received 4 March 2008; revision received 7 May 2008.

* Postal address: Department of Mathematics, Statistics and Computer Science, University of Illinois at Chicago (M/C 249), 851 South Morgan Street, Chicago, IL 60607-7045, USA. Email address: kness1@uic.edu

** Postal address: Department of Mathematics, State University of New York at New Paltz, 1 Hawk Dr. Suite 9, New Paltz, NY 12561-2443, USA. Email address: dominicd@ newpaltz.edu 
If the buffer has emptied at time $t$, it remains empty as long as the drift is negative. We let $\eta[Z(t)]=r_{k}$, given that $Z(t)$ is in state $k \in \mathcal{N}$, where $\mathcal{N}$ denotes the state space (in case of a finite space $\mathcal{N}=\{0,1, \ldots, N\})$. We shall assume throughout that $r_{k} \neq 0$ for all states. We shall also assume that $r_{k}>0$ for at least one $k \in \mathcal{N}$, since otherwise, in the steady state, the buffer is always empty.

We denote by $p_{k}$ the stationary probabilities of the birth-death process. When the capacity of the buffer is infinitely large, in order that a stationary distribution for $X(t)$ exists, the mean drift $\sum_{k \in \mathcal{N}} p_{k} r_{k}$ should be negative. We let

$$
\mathcal{N}^{+}=\left\{k \in \mathcal{N} \mid r_{k}>0\right\} \quad \text { and } \quad \mathcal{N}^{-}=\left\{k \in \mathcal{N} \mid r_{k}<0\right\}
$$

with $\mathcal{N}^{+} \cup \mathcal{N}^{-}=\mathcal{N}$, since we assume that the drift in each state is nonzero.

Setting

$$
P_{k}(t, x)=\operatorname{Pr}[X(t) \leq x, Z(t)=k], \quad t, x \geq 0, k \in \mathcal{N},
$$

the Kolmogorov forward equations for the Markov process $[X(t), Z(t)]$ are given by

$$
\frac{\partial P_{k}}{\partial t}+r_{k} \frac{\partial P_{k}}{\partial x}=\lambda_{k-1} P_{k-1}+\mu_{k+1} P_{k+1}-\left(\lambda_{k}+\mu_{k}\right) P_{k}, \quad k \in \mathcal{N} .
$$

For the stationary distribution $F_{k}(x)=\lim _{t \rightarrow \infty} P_{k}(t, x)$, we have

$$
r_{k} F_{k}^{\prime}=\lambda_{k-1} F_{k-1}+\mu_{k+1} F_{k+1}-\left(\lambda_{k}+\mu_{k}\right) F_{k}, \quad k \in \mathcal{N} .
$$

Since the buffer content is increasing whenever the drift is positive, the solution to (1) must satisfy the boundary conditions

$$
F_{k}(0)=0, \quad k \in \mathcal{N}^{+} .
$$

This means that there is no probability mass at $x=0$ if the drift takes you away from the boundary. Also, we must have $F_{k}(\infty)=p_{k}$ for $k \in \mathcal{N}$, as this is the marginal distribution of the regulating process $Z(t)$. The values of $F_{k}(0)$ for $k \in \mathcal{N}^{-}$are not a priori known. The 'half' boundary conditions, (2), make these problems difficult.

The purpose of this paper is to continue our asymptotic analysis of fluid models using the ray method [6], which we successfully applied in [4] to the model first studied by Anick et al. [2].

The paper is organized as follows. In Section 2 we state the basic equations. In Sections 3-7 we analyze these in various ranges of the state space $x \geq 0, k \geq 0$. In Section 8 we study the marginal distribution. Finally, in Section 9 we summarize and interpret the results.

\section{Problem statement}

Let $\mathcal{N}=\{0,1,2, \ldots\}$, and let the parameters $\lambda_{k}$ and $\mu_{k}$ be constant, with $\lambda_{k}=\lambda, \mu_{0}=0$, $\mu_{k}=\mu$ for $k \neq 0$, and

$$
\rho=\frac{\lambda}{\mu}<1
$$

The drift is taken as $r_{k}=k-c$, where $c$ represents the output rate of the buffer. We assume $c$ to be a positive noninteger number. This model corresponds to a fluid model modulated by the standard M/M/1 queue.

The forward Kolmogorov equations for $F_{k}(x)$ are then

$$
\begin{gathered}
(k-c) F_{k}^{\prime}(x)=\lambda F_{k-1}(x)+\mu F_{k+1}(x)-(\lambda+\mu) F_{k}(x), \quad k \geq 0, \\
\mu F_{0}(x)=\lambda F_{-1}(x),
\end{gathered}
$$


with boundary conditions

$$
F_{k}(0)=0, \quad k \geq 1+\lfloor c\rfloor
$$

and limiting distribution

$$
F_{k}(\infty)=(1-\rho) \rho^{k}, \quad k \geq 0,
$$

where $\rho$ is as defined in (3). Here, (5) defines $F_{-1}(x)$ and this condition is equivalent to $-c F_{0}^{\prime}(x)=\mu F_{1}(x)-\lambda F_{0}(x)$. Since the buffer capacity is infinite, we need the stability condition

$$
\frac{\rho}{1-\rho}<c, \quad \text { or } \quad \rho<1-\frac{1}{c+1} .
$$

A related model, with $r_{0}=\rho_{0}<0$ and $r_{k}=\rho>0$, was studied in [1], [3], and [18], where a spectral representation of the solution was obtained. The same model was analyzed in [10] using continued fractions. The general case, with arbitrary $r_{k}, \mu_{k}$, and $\lambda_{k}$, was studied in [7] and [17] using a family of orthogonal polynomials. The fluid queue driven by a general Markovian process was analyzed in [13]. A numerical method was presented in [8]. The full transient solution was considered in [14].

We shall analyze this model directly by using the differential-difference equation, (4), satisfied by $F_{k}(x)$. After appropriate scalings of $k$ and $x$, we analyze this equation asymptotically for $c \rightarrow \infty$, using singular perturbation methods. We also carefully treat various boundary and corner regions of the state space $\{(x, k): x \geq 0, k \geq 0\}$, and indeed we show that their analysis is needed in order to obtain the asymptotic expansions away from the boundaries.

\section{The ray expansion}

To analyze problem (4)-(7) for large $c$, we introduce the scaled variables $y$ and $z$, with $x=c^{2} y, k=c z$, and $z, y=O(1)$. We define the function $G(y, z)$ and the small parameter $\varepsilon$ by

$$
F_{k}(x)=G\left(x \varepsilon^{2}, k \varepsilon\right)=G(y, z), \quad \varepsilon=c^{-1},
$$

and note that $F_{k \pm 1}(x)=G(y, z \pm \varepsilon)$.

Then (4) becomes the following equation for $G(y, z)$ :

$$
\varepsilon(z-1) \frac{\partial G}{\partial y}(y, z)=\lambda G(y, z-\varepsilon)+\mu G(y, z+\varepsilon)-(\lambda+\mu) G(y, z),
$$

and (6) implies that

$$
G(0, z)=0, \quad z>1 .
$$

Also, from (7) we have

$$
F_{k}(\infty)=G(\infty, z)=(1-\rho) \exp \left(\frac{1}{\varepsilon} z \ln (\rho)\right), \quad z>0 .
$$

To find $G(y, z)$ for small $\varepsilon$, we shall use the ray method. Thus, we consider solutions which have the asymptotic form

$$
G(y, z) \sim \varepsilon^{\nu} \exp \left(\frac{1}{\varepsilon} \Psi(y, z)\right) \mathbb{K}(y, z),
$$

where $v$ is a constant that must be determined. Using (10) in (8), with

$$
\frac{1}{\varepsilon} \Psi(y, z \pm \varepsilon)=\frac{1}{\varepsilon} \Psi \pm \Psi_{z}+\frac{1}{2} \Psi_{z z} \varepsilon+O\left(\varepsilon^{2}\right)
$$


dividing by $\exp ((1 / \varepsilon) \Psi(y, z))$, and expanding in powers of $\varepsilon$, we obtain the eikonal equation for $\Psi(y, z)$,

$$
\mu\left(1-\mathrm{e}^{q}\right)+\lambda\left(1-\mathrm{e}^{-q}\right)+(z-1) p=0,
$$

and the transport equation for $\mathbb{K}(y, z)$,

$$
\left(\mu \mathrm{e}^{q}-\lambda \mathrm{e}^{-q}\right) \frac{\partial \mathbb{K}}{\partial z}+(1-z) \frac{\partial \mathbb{K}}{\partial y}+\frac{1}{2} \frac{\partial q}{\partial z}\left(\mu \mathrm{e}^{q}+\lambda \mathrm{e}^{-q}\right) \mathbb{K}=0,
$$

where $p=\Psi_{y}=\partial \Psi / \partial y$ and $q=\Psi_{z}=\partial \Psi / \partial z$. To solve (11) and (12), we use the method of characteristics, which we briefly review below.

Given the first-order partial differential equation $\mathfrak{F}(y, z, \Psi, p, q)=0$, we search for a solution $\Psi(y, z)$ by solving the following system of 'characteristic equations':

$$
\begin{aligned}
\dot{y}=\frac{\partial y}{\partial t} & =\mathfrak{F}_{p}, \quad \dot{z}=\mathfrak{F}_{q}, \quad \dot{p}=-\mathfrak{F}_{y}-p \mathfrak{F}_{\Psi}, \\
\dot{q} & =-\mathfrak{F}_{z}-q \mathfrak{F}_{\Psi}, \quad \dot{\psi}=p \mathfrak{F}_{p}+q \mathfrak{F}_{q},
\end{aligned}
$$

where we now consider $\{y, z, \psi, p, q\}$ to all be functions of the variables $s$ and $t$, with $\psi(s, t)=$ $\Psi(y, z)$. Here $t$ measures how far we are along a particular characteristic curve or ray and $s$ indexes them.

For the eikonal equation, (11), the characteristic equations are

$$
\begin{aligned}
\dot{y} & =z-1, \\
\dot{z} & =\lambda \mathrm{e}^{-q}-\mu \mathrm{e}^{q}, \\
\dot{p} & =0, \\
\dot{q} & =-p, \\
\dot{\psi} & =p(z-1)+q\left(\lambda \mathrm{e}^{-q}-\mu \mathrm{e}^{q}\right) .
\end{aligned}
$$

The particular solution is determined by the initial conditions at $t=0$. We shall show that, for this problem, two different types of solutions are needed; these correspond to two distinct families of rays.

Setting $\left.p\right|_{t=0}=s$ and $\left.q\right|_{t=0}=B$, and solving (13c)-(13d) yields

$$
p=s, \quad q=B-s t,
$$

so that $p$ is constant along a ray.

\subsection{The rays from $(0,1)$}

We now consider the family of rays emanating from the point $y=0, z=1$. Evaluating (11) at $t=0$ we obtain

$$
\mu\left(1-\mathrm{e}^{B}\right)+\lambda\left(1-\mathrm{e}^{-B}\right)=0,
$$

so that $B=0$ or $B=\ln (\rho)$. From (13b) and (14), with the initial condition $z(s, 0)=1$, we obtain

$$
z=\frac{1}{s}\left(\lambda \mathrm{e}^{-B}\left(\mathrm{e}^{s t}-1\right)+\mu \mathrm{e}^{B}\left(\mathrm{e}^{-s t}-1\right)\right)+1 .
$$

From (13a) we have

$$
\dot{y}(s, 0)=z(s, 0)-1=0
$$




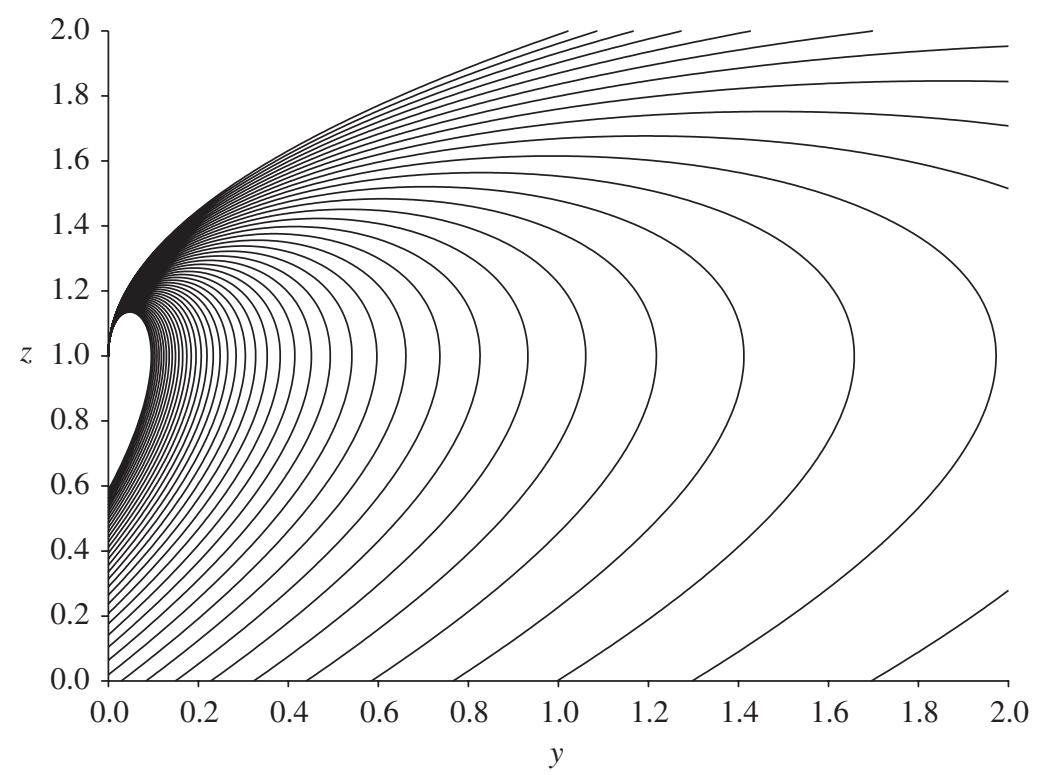

FIGURE 1: A sketch of the rays from $(0,1)$.

and

$$
\ddot{y}(s, 0)=\dot{z}(s, 0)=\lambda \mathrm{e}^{-B}-\mu \mathrm{e}^{B} .
$$

Thus, we have

$$
\ddot{y}(s, 0)= \begin{cases}\lambda-\mu<0, & B=0, \\ \mu-\lambda>0, & B=\ln (\rho) .\end{cases}
$$

Using the initial condition $y(s, 0)=0$ and expanding in powers of $t$, we obtain

$$
y(s, t) \sim \ddot{y}(s, 0) \frac{t^{2}}{2}, \quad t \rightarrow 0,
$$

and in order to have $y>0$ for $t>0$ (i.e. for the rays to enter the domain $[0, \infty) \times[0, \infty)$ ), we need to choose $B=\ln (\rho)$ with $B<0$, since $\rho<1$. Integrating (13a) and using (15), we conclude that

$$
\begin{gathered}
y(s, t)=\frac{1}{s^{2}}\left(\mu\left(\mathrm{e}^{s t}-s t-1\right)+\lambda\left(1-s t-\mathrm{e}^{-s t}\right)\right), \\
z(s, t)=\frac{1}{s}\left(\mu\left(\mathrm{e}^{s t}-1\right)+\lambda\left(\mathrm{e}^{-s t}-1\right)\right)+1 .
\end{gathered}
$$

This yields the rays that emanate from $(0,1)$ in parametric form. Several rays are sketched in Figure 1.

For $t \geq 0$ and each value of $s,(16)$ and (17) determine a ray in the $(y, z)$-plane, which starts from $(0,1)$ at $t=0$. We discuss a particular ray which can be obtained in an explicit form. For $s=0$, we can eliminate $t$ from (17) and obtain

$$
y=Y_{0}(z):=\frac{(z-1)^{2}}{2(\mu-\lambda)}, \quad s=0, z \geq 1,
$$


and along this ray, $t$ and $z$ are related by

$$
t\left(Y_{0}, z\right)=T_{0}(z)=\frac{z-1}{\mu-\lambda}, \quad s=0, z \geq 1 .
$$

For $s>0$, we have both $y(s, t)$ and $z(s, t)$ increasing for $t>0$. For $s<0$, the rays reach a maximum value in $z$ at $t=T_{1}$, where

$$
T_{1}(s)=\frac{1}{2 s} \ln (\rho), \quad s<0,
$$

and we have

$$
\begin{gathered}
y\left(s, T_{1}\right)=\frac{1}{s^{2}}\left(\lambda-\mu-\frac{1}{2}(\lambda+\mu) \ln (\rho)\right), \\
z\left(s, T_{1}\right)=\frac{1}{s}(2 \sqrt{\lambda \mu}-(\lambda+\mu))+1 .
\end{gathered}
$$

From (13a) we see that the maximum value in $y$ is achieved at the same time that $z=1$, and that occurs at $t=T_{2}$ with

$$
T_{2}(s)=\frac{1}{s} \ln (\rho), \quad s<0,
$$

and

$$
y\left(s, T_{2}\right)=\frac{1}{s^{2}}(2(\lambda-\mu)-(\lambda+\mu) \ln (\rho)) .
$$

Inverting (16)-(17), we can write $s=S(y, z), t=T(y, z)$, and

$$
\Psi(y, z)=\psi[S(y, z), T(y, z)], \quad \mathbb{K}(y, z)=K[S(y, z), T(y, z)] .
$$

We will use this notation in the rest of the article.

\subsection{The functions $\Psi$ and $\mathbb{K}$}

From (13e) we have

$$
\dot{\psi}=\mu \mathrm{e}^{s t}(1+\ln (\rho)-t s)+\lambda \mathrm{e}^{-s t}(1-\ln (\rho)+t s),
$$

which we can integrate to obtain

$$
\begin{gathered}
\psi(s, t)=\frac{\mu}{s} \mathrm{e}^{s t}(2+\ln (\rho)-t s)-\frac{\lambda}{s} \mathrm{e}^{-s t}(2-\ln (\rho)+t s) \\
+\psi(s, 0)-\frac{\mu}{s}(2+\ln (\rho))+\frac{\lambda}{s}(2-\ln (\rho)) .
\end{gathered}
$$

Obviously, $\psi(s, 0) \equiv \psi_{0}$ is a constant, since all rays start at the same point. Setting $s=0$ in (20) and using (19), we obtain $\psi(0, t)=\psi_{0}+(z-1) \ln (\rho)$ and, therefore, taking the limit as $t \rightarrow \infty$, we obtain $\Psi(\infty, z)=\psi_{0}+(z-1) \ln (\rho)$. On the other hand, from (9) we have $\Psi(\infty, z)=z \ln (\rho)$ and we conclude that $\psi_{0}=\ln (\rho)$.

Solving for $\mathrm{e}^{s t}$ in (16)-(17), we obtain

$$
\mathrm{e}^{s t}=1+\frac{s}{2 \mu}(z-1+y s+t(\lambda+\mu)), \quad \mathrm{e}^{-s t}=1+\frac{s}{2 \lambda}(z-1-y s-t(\lambda+\mu)) .
$$


Replacing (21) in (20), we obtain

$$
\psi=2 y s+(\ln (\rho)-s t)(z-1)+\ln (\rho) .
$$

We shall now solve the transport equation, (12), which we rewrite as

$$
(z-1) \frac{\partial \mathbb{K}}{\partial y}+\left(\lambda \mathrm{e}^{-q}-\mu \mathrm{e}^{q}\right) \frac{\partial \mathbb{K}}{\partial z}=\frac{1}{2} \frac{\partial q}{\partial z}\left(\mu \mathrm{e}^{q}+\lambda \mathrm{e}^{-q}\right) \mathbb{K} .
$$

Using (16) and (17) in (23), we have

$$
\frac{\partial K}{\partial t}=\frac{1}{2} \frac{\partial q}{\partial z}\left(\mu \mathrm{e}^{q}+\lambda \mathrm{e}^{-q}\right) K .
$$

To solve (24), we need to compute $\partial q / \partial z$ as a function of $s$ and $t$. Use of the chain rule gives

$$
\left[\begin{array}{ll}
\frac{\partial t}{\partial y} & \frac{\partial t}{\partial z} \\
\frac{\partial s}{\partial y} & \frac{\partial s}{\partial z}
\end{array}\right]=\frac{1}{J}\left[\begin{array}{cc}
\frac{\partial z}{\partial s} & -\frac{\partial y}{\partial s} \\
-\frac{\partial z}{\partial t} & \frac{\partial y}{\partial t}
\end{array}\right]
$$

where the Jacobian $J(s, t)$ is defined by

$$
J(s, t)=\frac{\partial y}{\partial t} \frac{\partial z}{\partial s}-\frac{\partial y}{\partial s} \frac{\partial z}{\partial t}=\frac{1}{s}\left(2 \frac{\partial z}{\partial t} y-(z-1)^{2}\right) .
$$

Using (25), we can show after some algebra that

$$
\frac{\partial q}{\partial z}=-\frac{2 y}{J}
$$

while (21) gives

$$
\mu \mathrm{e}^{q}+\lambda \mathrm{e}^{-q}=\mu+\lambda+(z-1) s .
$$

Thus, the transport equation, (24), becomes

$$
\frac{1}{K} \frac{\partial K}{\partial t}=-\frac{y}{J}(\mu+\lambda+(z-1) s) .
$$

Using (16) and (17) in (26), we have

$$
\frac{\partial J}{\partial t}=\frac{2 y}{s} \frac{\partial^{2} z}{\partial t^{2}}=2 y(\mu+\lambda+(z-1) s) .
$$

Combining (28) and (29), we obtain

$$
\frac{1}{K} \frac{\partial K}{\partial t}=-\frac{1}{2 J} \frac{\partial J}{\partial t}
$$

whose solution is

$$
K(s, t)=\frac{K_{0}(s)}{\sqrt{J(s, t)}},
$$

where $K_{0}(s)$ is a function to be determined. 
From (26) we have

$$
J(s, t)=\left(\mu^{2}-\lambda^{2}\right) \frac{t^{3}}{3}+O\left(t^{4}\right), \quad t \rightarrow 0 .
$$

Since the Jacobian vanishes as $t \rightarrow 0$, the ray expansion ceases to be valid near the point $(0,1)$, where a separate analysis is needed.

So far we have determined the exponent $\psi(s, t)$ and the leading amplitude $K(s, t)$ except for the function $K_{0}(s)$ in (30) and the power $v$ in (10). In Section 4 we will determine them by matching (10) to a corner layer solution valid in a neighborhood of the point $(0,1)$.

\subsection{The rays from $\infty$}

Denoting the domain in the $(y, z)$-plane by

$$
\mathfrak{D}=[0, \infty) \times[0, \infty),
$$

we must determine what part of $\mathfrak{D}$ the rays from $\infty$ fill. The expansion corresponding to these rays must satisfy boundary condition (9). Thus, we have

$$
p(\infty, z)=p_{\infty}=0, \quad q(\infty, z)=q_{\infty}=\ln (\rho),
$$

while (13a)-(13b) yield equations for the rays $y_{\infty}(t), z_{\infty}(t)$,

$$
\dot{y}_{\infty}=z_{\infty}-1, \quad \text { and } \quad \dot{z}_{\infty}=\mu-\lambda,
$$

or, eliminating $t$ from system (32) and writing $y_{\infty}(t)=Y_{\infty}(z)$, we obtain

$$
\frac{\mathrm{d} Y_{\infty}}{\mathrm{d} z}=\frac{z-1}{\mu-\lambda} .
$$

Solving (32) subject to the initial condition $Y_{\infty}\left(z_{0}\right)=y_{0}$, where $y_{0} \times z_{0}=0$, we obtain

$$
Y_{\infty}(z)=y_{0}+\frac{1}{2(\mu-\lambda)}\left((z-1)^{2}-\left(z_{0}-1\right)^{2}\right) .
$$

From (33), it follows that the minimum value in $y$ occurs when $z=1$. Hence, for $y$ to be positive, we must have

$$
y_{0}>\frac{\left(z_{0}-1\right)^{2}}{2(\mu-\lambda)}=Y_{0}\left(z_{0}\right)
$$

where $Y_{0}(z)$ is as defined in (18). Therefore, the rays from $\infty$ fill the region given by

$$
R=\{0 \leq y, 0 \leq z \leq 1\} \cup\left\{Y_{0}(z) \leq y, 1 \leq z\right\} .
$$

The complementary region,

$$
R^{\mathrm{C}}=\left\{0 \leq y<Y_{0}(z), 1 \leq z\right\}
$$

is a shadow of the rays from $\infty$. In $R^{\mathrm{C}}, G$ is given by $(10)$, as only the rays from $(0,1)$ are present (see Figure 2). In region $R$, both the rays coming from $(0,1)$ and the rays coming from $\infty$ must be taken into account. We add (9) and (10) to represent $G$ in the asymptotic form

$$
G(y, z) \sim(1-\rho) \exp \left(\frac{1}{\varepsilon} z \ln (\rho)\right)+\varepsilon^{\nu} \exp \left(\frac{1}{\varepsilon} \Psi(y, z)\right) \mathbb{K}(y, z), \quad(y, z) \in R .
$$

We can show that $z \ln (\rho)>\Psi(y, z)$ in the interior of $R$, so that $G(y, z) \sim G(\infty, z)$. However, in $R$ we can write (35) as $G(y, z)-G(\infty, z) \sim \varepsilon^{v} \exp ((1 / \varepsilon) \Psi(y, z)) \mathbb{K}(y, z)$. 


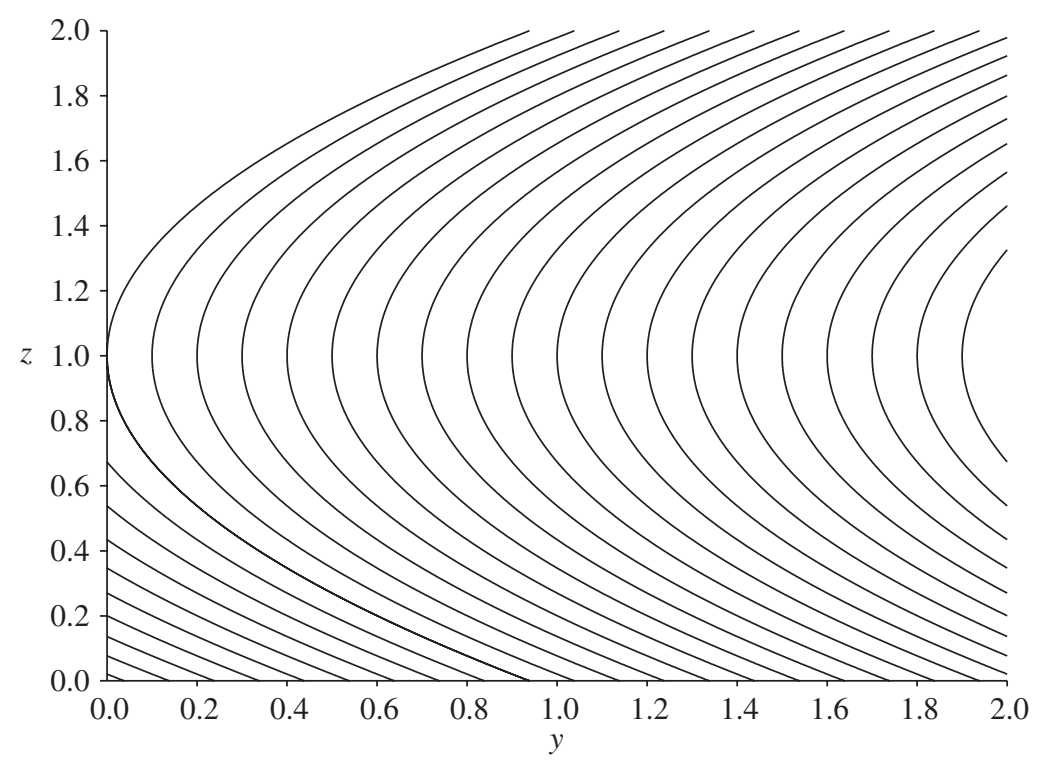

FIGURE 2: A sketch of the rays from $\infty$.

\section{The corner layer at $(0,1)$}

We determine the constant $v$ in (10) and the function $K_{0}(s)$ in (30) by considering carefully the region where the rays from $(0,1)$ enter the domain $\mathfrak{D}$, and using asymptotic matching. We introduce the stretched variable $l$, the function $F_{l}^{(1)}$, and the parameter $\alpha$ defined by

$$
\begin{gathered}
F_{k}(x)=G_{1}(x, k-c+\alpha)=F_{l}^{(1)}(x), \\
l=k-c+\alpha, \quad-\infty<l<\infty, \quad \alpha=c-\lfloor c\rfloor, \quad 0<\alpha<1 .
\end{gathered}
$$

Note that $\alpha$ is the fractional part of $c$ and $l$ takes on integer values. Use of (36) in (4) yields the equation

$$
(l-\alpha) \frac{\mathrm{d} F_{l}^{(1)}}{\mathrm{d} x}=\mu F_{l+1}^{(1)}+\lambda F_{l-1}^{(1)}-(\lambda+\mu) F_{l}^{(1)}, \quad x>0, l \in \mathbb{Z} .
$$

Also, (6) gives the boundary condition

$$
F_{l}^{(1)}(0)=0, \quad l \geq 1,
$$

and (9) implies that $F_{k}(\infty)=F_{l}^{(1)}(\infty)$ with

$$
F_{l}^{(1)}(\infty)=(1-\rho) \rho^{l-\alpha} \exp \left(\frac{1}{\varepsilon} \ln (\rho)\right) .
$$

For a fixed $l$ and $x \rightarrow \infty$, we approach the interior of $R$, where (35) applies. Thus, (39) is the asymptotic matching condition between the corner layer and the solution in $R$. We shall examine the matching to $R^{\mathrm{C}}$ later.

Since problem (37)-(39) is of interest by itself, we solve it in a slightly more general setting in the next theorem. 
Theorem 1. Suppose that the function $\Phi_{l}(x)$ satisfies the equation

$$
(l-\alpha) \frac{\mathrm{d} \Phi_{l}}{\mathrm{~d} x}=A \Phi_{l+1}+C \Phi_{l-1}-B \Phi_{l}, \quad x>0, l \in \mathbb{Z},
$$

with

$$
B>2 \sqrt{A C}=\beta>0
$$

and the boundary conditions

$$
\begin{gathered}
\Phi_{l}(0)=0, \quad l \geq 1, \\
\Phi_{l}(\infty)=\mu_{\infty} r^{l}, \quad l \in \mathbb{Z},
\end{gathered}
$$

where

$$
A r^{2}-B r+C=0
$$

Then, $\Phi_{l}$ has the integral representation

$$
\begin{aligned}
\Phi_{l}(x)= & \mu_{\infty}\left(\frac{C}{A}\right)^{l / 2} \sqrt{\frac{\Delta}{B}} \\
\times \frac{1}{2 \pi \mathrm{i}} \int_{\mathrm{Br}} & \exp \left(x \theta+\frac{B-\Delta}{\theta}\right) \frac{1}{\theta} \Gamma\left(1-\alpha+\frac{B}{\theta}\right) \\
& \times J_{l-\alpha+B / \theta}\left(\frac{\beta}{\theta}\right)\left(\frac{B-\Delta}{\beta} \frac{B}{\theta}\right)^{\alpha-B / \theta} \mathrm{d} \theta
\end{aligned}
$$

and the spectral representation

$$
\begin{aligned}
\Phi_{l}(x)= & \mu_{\infty} r^{l} \\
-\mu_{\infty}\left(\frac{C}{A}\right)^{l / 2} \sqrt{\frac{\Delta}{B}} \sum_{j=0}^{\infty} & \frac{(j+1-\alpha)^{j}}{j !}\left(\frac{B-\Delta}{\beta}\right)^{j+1} \\
& \times \exp \left(x \theta_{j}+\frac{B-\Delta}{\theta_{j}}\right) J_{l-j-1}\left(\frac{\beta}{\theta_{j}}\right),
\end{aligned}
$$

where $\mathrm{Br}$ is a vertical contour in the complex $\theta$-plane on which $\operatorname{Re}(\theta)>0, \Gamma(\cdot)$ denotes the gamma function, $J_{v}(\cdot)$ is the Bessel function of the first kind,

$$
\Delta=\sqrt{B^{2}-\beta^{2}}=\sqrt{B^{2}-4 A C},
$$

and

$$
\theta_{j}=-\frac{B}{j+1-\alpha}, \quad j=0,1, \ldots
$$

Proof. Equation (40) admits the separable solutions

$$
\Phi_{l}(x)=\mathrm{e}^{\theta x} h_{l}(\theta)
$$

if $h_{l}(\theta)$ satisfies the difference equation

$$
A h_{l+1}+C h_{l-1}=((l-\alpha) \theta+B) h_{l} .
$$


Setting

$$
h_{l}(\theta)=\left(\frac{C}{A}\right)^{l / 2} H_{l}(\theta)
$$

we see that

$$
H_{l+1}+H_{l-1}=\frac{2}{\beta}((l-\alpha) \theta+B) H_{l} .
$$

The only solutions to (46) which have acceptable behavior as $l \rightarrow \infty$ are of the form

$$
H_{l}(\theta)=J_{l-\alpha+B / \theta}\left(\frac{\beta}{\theta}\right) .
$$

If (45) is not to grow as $x \rightarrow \infty$, we need $\theta \leq 0$. But, except when $v$ is an integer, the Bessel function $J_{v}(\cdot)$ is complex for negative argument. Therefore, we need

$$
-\alpha+\frac{B}{\theta}=-1,-2, \ldots
$$

or

$$
\theta_{j}=-\frac{B}{j+1-\alpha}<0, \quad j \geq 0 .
$$

It follows that the general solution to (40) takes the form

$$
\Phi_{l}(x)=\Phi_{l}(\infty)+\left(\frac{C}{A}\right)^{l / 2} \sum_{j \geq 0} a_{j} \exp \left(\theta_{j} x\right) J_{l-\alpha+B / \theta_{j}}\left(\frac{\beta}{\theta_{j}}\right)
$$

or

$$
\Phi_{l}(x)=\mu_{\infty} r^{l}+\left(\frac{C}{A}\right)^{l / 2} \sum_{j \geq 0} a_{j} \exp \left(-\frac{B}{j+1-\alpha} x\right) J_{l-1-j}\left(-\frac{\beta}{B}(j+1-\alpha)\right),
$$

where the coefficients $a_{j}$ in the above (spectral) representation remain to be determined. Taking the Laplace transform

$$
\hat{\Phi}_{l}(\theta)=\int_{0}^{\infty} \mathrm{e}^{-\theta x} \Phi_{l}(x) \mathrm{d} x
$$

of (48) we obtain

$$
\hat{\Phi}_{l}(\theta)=\mu_{\infty} r^{l} \frac{1}{\theta}+\left(\frac{C}{A}\right)^{l / 2} \sum_{j \geq 0} \frac{a_{j}}{\theta+B /(j+1-\alpha)} J_{j+1-l}\left(\frac{\beta}{B}(j+1-\alpha)\right) .
$$

Thus, the only singularities of $\hat{\Phi}_{l}(\theta)$ are simple poles at $\theta=0$ and $\theta=\theta_{j}, j \geq 0$. It is well known that the gamma function $\Gamma(z)$ has simple poles at $z=0,-1,-2, \ldots$. Hence, we shall represent $\hat{\Phi}_{l}(\theta)$ as

$$
\hat{\Phi}_{l}(\theta)=\left(\frac{C}{A}\right)^{l / 2} \frac{1}{\theta} \Gamma\left(\frac{B}{\theta}+1-\alpha\right) J_{l-\alpha+B / \theta}\left(\frac{\beta}{\theta}\right) f(\theta),
$$

where $f(\theta)$ is chosen such that

$$
\Gamma\left(\frac{B}{\theta}+1-\alpha\right) J_{l-\alpha+B / \theta}\left(\frac{\beta}{\theta}\right) f(\theta)
$$


is analytic for $\operatorname{Re}(\theta)>-B /(1-\alpha)$. Taking the Laplace transform in (40), we obtain the equation

$$
(l-\alpha) \theta \hat{\Phi}_{l}(\theta)=A \hat{\Phi}_{l+1}(\theta)+C \hat{\Phi}_{l-1}(\theta)-B \hat{\Phi}_{l}(\theta), \quad l \geq 1,
$$

which is satisfied by (49). By the inversion formula for the Laplace transform, we have

$$
\Phi_{l}(x)=\left(\frac{C}{A}\right)^{l / 2} \frac{1}{2 \pi \mathrm{i}} \int_{\mathrm{Br}} \mathrm{e}^{x \theta} \frac{1}{\theta} \Gamma\left(\frac{B}{\theta}+1-\alpha\right) J_{l-\alpha+B / \theta}\left(\frac{\beta}{\theta}\right) f(\theta) \mathrm{d} \theta .
$$

Since the residue of $\hat{\Phi}_{l}(\theta)$ at $\theta=0$ corresponds to $\Phi_{l}(\infty)$, we must have

$$
\left(\frac{C}{A}\right)^{l / 2} \Gamma\left(\frac{B}{\theta}+1-\alpha\right) J_{l-\alpha+B / \theta}\left(\frac{\beta}{\theta}\right) f(\theta) \rightarrow \mu_{\infty} r^{l} \quad \text { as } \theta \rightarrow 0 .
$$

Using the asymptotic formulae $(z \rightarrow \infty)$

$$
\Gamma(a+b z) \sim \sqrt{2 \pi} \mathrm{e}^{-b z}(b z)^{a+b z-1 / 2}, \quad b>0,
$$

and

$$
J_{a+b z}(c z) \sim \frac{1}{\sqrt{2 \pi z}\left(b^{2}-c^{2}\right)^{1 / 4}} \exp \left(z \sqrt{b^{2}-c^{2}}\right)\left(\frac{b-\sqrt{b^{2}-c^{2}}}{c}\right)^{a+b z}, \quad b>c>0,
$$

in (49), we see that

$$
\Gamma\left(\frac{B}{\theta}+1-\alpha\right) J_{l-\alpha+B / \theta}\left(\frac{\beta}{\theta}\right) \sim \mathrm{e}^{(\Delta-B) / \theta}\left(\frac{B-\Delta}{\beta}\right)^{l}\left(\frac{B-\Delta}{\beta} \frac{B}{\theta}\right)^{B / \theta-\alpha} \sqrt{\frac{B}{\Delta}}, \quad \theta \rightarrow 0,
$$

or, using (42),

$$
\Gamma\left(\frac{B}{\theta}+1-\alpha\right) J_{l-\alpha+B / \theta}\left(\frac{\beta}{\theta}\right) \sim \mathrm{e}^{(\Delta-B) / \theta} r^{l}\left(\frac{A}{C}\right)^{l / 2}\left(\frac{B-\Delta}{\beta} \frac{B}{\theta}\right)^{B / \theta-\alpha} \sqrt{\frac{B}{\Delta}}, \quad \theta \rightarrow 0 .
$$

Therefore, we write

$$
f(\theta)=\mu_{\infty} \sqrt{\frac{\Delta}{B}} \exp (\Upsilon(\theta)) \tilde{f}(\theta)
$$

where

$$
\Upsilon(\theta)=\frac{B-\Delta}{\theta}-\left(\frac{B}{\theta}-\alpha\right) \ln \left(\frac{B-\Delta}{\beta} \frac{B}{\theta}\right),
$$

and $\tilde{f}(\theta)$ is entire, with $\tilde{f}(0)=1$. By combining the preceding results we have

$$
\Phi_{l}(x)=\mu_{\infty} \sqrt{\frac{\Delta}{B}}\left(\frac{C}{A}\right)^{l / 2} \frac{1}{2 \pi \mathrm{i}} \int_{\mathrm{Br}} \mathrm{e}^{x \theta} \frac{1}{\theta} \Gamma\left(\frac{B}{\theta}+1-\alpha\right) J_{l-\alpha+B / \theta}\left(\frac{\beta}{\theta}\right) \exp (\Upsilon(\theta)) \tilde{f}(\theta) \mathrm{d} \theta .
$$

Boundary condition (41) implies that

$$
\lim _{\theta \rightarrow \infty}\left[\theta \hat{\Phi}_{l}(\theta)\right]=0, \quad l \geq 1,
$$

and using the asymptotic formula

$$
J_{v}(z) \sim\left(\frac{z}{2}\right)^{v} \frac{1}{\Gamma(v+1)}, \quad z \rightarrow 0, v \neq-1,-2, \ldots
$$


in (53), we have

$$
\begin{aligned}
& \frac{1}{\theta} \Gamma\left(\frac{B}{\theta}+1-\alpha\right) J_{l-\alpha+B / \theta}\left(\frac{2 \beta}{\theta}\right) \exp (\Upsilon(\theta)) \\
& \quad \sim\left(\frac{1}{\theta}\right)^{l+1} \frac{\Gamma(1-\alpha)}{\Gamma(l-\alpha+1)}\left(\frac{\beta}{2}\right)^{l}\left(\frac{r B}{C}\right)^{\alpha}, \quad \theta \rightarrow \infty
\end{aligned}
$$

Setting $l=1$, we obtain

$$
\tilde{f}(\theta)=o(\theta), \quad \theta \rightarrow \infty,
$$

and Liouville's theorem forces $\tilde{f}(\theta)$ to be a constant. Since $\tilde{f}(0)=1$, we have $\tilde{f}(\theta) \equiv 1$. Thus, (53) becomes

$$
\begin{aligned}
\Phi_{l}(x)= & \mu_{\infty} \sqrt{\frac{\Delta}{B}}\left(\frac{C}{A}\right)^{l / 2} \\
& \times \frac{1}{2 \pi \mathrm{i}} \int_{\mathrm{Br}}\left(\mathrm{e}^{x \theta} \frac{1}{\theta} \Gamma\left(\frac{B}{\theta}+1-\alpha\right) J_{l-\alpha+B / \theta}\left(\frac{\beta}{\theta}\right) \exp (\Upsilon(\theta))\right) \mathrm{d} \theta,
\end{aligned}
$$

and we obtain (43). The coefficients $a_{j}$ in spectral expansion (48) are determined from (54) by applying the residue theorem. Noting that

$$
\operatorname{Res}\left[\frac{1}{\theta} \Gamma\left(\frac{B}{\theta}+1-\alpha\right), \theta=\theta_{j}\right]=\frac{(-1)^{j}}{(j+1-\alpha) j !},
$$

we obtain

$$
a_{j}=\mu_{\infty} \sqrt{\frac{\Delta}{B}}\left(\frac{C}{A}\right)^{l / 2} \exp \left(x \theta_{j}\right) \frac{(-1)^{j}}{(j+1-\alpha) j !} J_{l-\alpha+B / \theta_{j}}\left(\frac{\beta}{\theta_{j}}\right) \exp \left(\Upsilon\left(\theta_{j}\right)\right), \quad j \geq 0 .
$$

Using (47) in (52), we obtain

$$
\exp \left(\Upsilon\left(\theta_{j}\right)\right)=\exp \left(\frac{B-\Delta}{\theta_{j}}\right)\left(\frac{B-\Delta}{\beta}\right)^{j+1}(\alpha-j-1)^{j+1},
$$

and (44) follows.

For the problem (37)-(39), we have

$$
\begin{gathered}
A=\mu, \quad B=\lambda+\mu, \quad C=\lambda, \quad r=\rho, \\
\mu_{\infty}=(1-\rho) \rho^{c-\alpha}, \quad \Delta=\mu-\lambda,
\end{gathered}
$$

and, therefore,

$$
\begin{aligned}
F_{l}^{(1)}(x)=(1-\rho) \sqrt{\frac{\mu-\lambda}{\mu+\lambda}} \rho^{c-\alpha+l / 2} \\
\times \frac{1}{2 \pi \mathrm{i}} \int_{\mathrm{Br}}\left(\mathrm{e}^{x \theta} \frac{1}{\theta} \Gamma\left(\frac{\lambda+\mu}{\theta}+1-\alpha\right)\right. \\
\quad \times J_{\left.l-\alpha+(\lambda+\mu) / \theta\left(\frac{2 \sqrt{\mu \lambda}}{\theta}\right) \exp (\Lambda(\theta))\right) \mathrm{d} \theta}
\end{aligned}
$$


where

$$
\Lambda(\theta)=\frac{2 \lambda}{\theta}-\left(\frac{\lambda+\mu}{\theta}-\alpha\right) \ln \left(\sqrt{\rho} \frac{\lambda+\mu}{\theta}\right) .
$$

Also,

$$
\begin{aligned}
& F_{l}^{(1)}(x)=(1-\rho) \rho^{c-\alpha+l / 2} \\
& \times\left(\rho^{l / 2}-\sqrt{\frac{\mu-\lambda}{\mu+\lambda}} \sum_{j=0}^{\infty} \frac{(j+1-\alpha)^{j}}{j !}\right. \\
& \left.\times \rho^{(j+1) / 2} \exp \left(x \vartheta_{j}+\frac{2 \lambda}{\vartheta_{j}}\right) J_{l-j-1}\left(\frac{2 \sqrt{\mu \lambda}}{\vartheta_{j}}\right)\right),
\end{aligned}
$$

where

$$
\vartheta_{j}=-\frac{\lambda+\mu}{j+1-\alpha}, \quad j \geq 0 .
$$

This completes the determination of the spectral and integral representations of $F_{l}^{(1)}(x)$ and, hence, the leading term for $F_{k}(x)$ in the corner region.

\subsection{Matching the corner and $R^{\mathrm{C}}$ regions}

In this subsection we shall determine the function $K_{0}(s)$ in (30) and the power $v$ in (10). We begin with the following result.

Theorem 2. With the same hypothesis and notation as in Theorem 1, let $\Omega$ be defined by

$$
\Omega=\frac{2 \Delta x}{(l-\alpha)^{2}}=O(1) .
$$

Then,

$$
\Phi_{l}(x) \sim \mu_{\infty} r^{l} \sqrt{\frac{2 B}{3 \pi \Delta(l-\alpha)}}(1-\Omega)^{-1}, \quad x \rightarrow \infty, l \rightarrow \infty, \Omega \rightarrow 1 .
$$

Proof. We set

$$
\theta=\varepsilon \Theta, \quad \eta=(l-\alpha) \theta+B=(z-1) \Theta+B, \quad \eta, \Theta=O(1), \eta, \Theta>0,
$$

and using (51), we obtain

$$
\begin{aligned}
J_{\eta /(\varepsilon \Theta)}\left(\frac{\beta}{\varepsilon \Theta}\right) & \sim \frac{\sqrt{\varepsilon \Theta}}{\sqrt{2 \pi}\left(\eta^{2}-\beta^{2}\right)^{1 / 4}} \exp \left(\frac{1}{\varepsilon \Theta} \sqrt{\eta^{2}-\beta^{2}}\right)\left(\frac{\eta-\sqrt{\eta^{2}-\beta^{2}}}{\beta}\right)^{\eta /(\varepsilon \Theta)} \\
& =\sqrt{\frac{\varepsilon \Theta}{2 \pi p(\eta)}} \exp \left(\frac{1}{\varepsilon \Theta}\left(p(\eta)+\eta \ln \left(\frac{\eta-p(\eta)}{\beta}\right)\right)\right), \quad \varepsilon \rightarrow 0,
\end{aligned}
$$

where

$$
p(\eta)=\sqrt{\eta^{2}-\beta^{2}}, \quad p(B)=\Delta .
$$

Use of (50) gives

$$
\Gamma\left(\frac{B}{\varepsilon \Theta}+1-\alpha\right) \sim \sqrt{2 \pi} \exp \left(\frac{B}{\varepsilon \Theta}\left(\ln \left(\frac{B}{\varepsilon \Theta}\right)-1\right)\right)\left(\frac{B}{\varepsilon \Theta}\right)^{1 / 2-\alpha},
$$


and from (52) we have

$$
\exp (\Upsilon(\varepsilon \Theta))=\exp \left(\frac{B-\Delta}{\varepsilon \Theta}-\frac{B}{\varepsilon \Theta} \ln \left(\frac{B-\Delta}{\beta} \frac{B}{\theta}\right)\right)\left(\frac{B-\Delta}{\beta} \frac{B}{\varepsilon \Theta}\right)^{\alpha} .
$$

Therefore,

$$
\begin{aligned}
J_{\eta /(\varepsilon \Theta)} & \left(\frac{\beta}{\varepsilon \Theta}\right) \Gamma\left(\frac{B}{\varepsilon \Theta}+1-\alpha\right) \exp (\Upsilon(\varepsilon \Theta)) \\
& \sim \sqrt{\frac{B}{p(\eta)}}\left(\frac{B-\Delta}{\beta}\right)^{\alpha} \exp \left(\frac{1}{\varepsilon \Theta}\left(p(\eta)+\eta \ln \left(\frac{\eta-p(\eta)}{\beta}\right)-\Delta-B \ln \left(\frac{B-\Delta}{\beta}\right)\right)\right) .
\end{aligned}
$$

Using (59) in (54) yields, in terms of $z$ and $\Omega$,

$$
\Phi_{l}(x) \sim \mu_{\infty} r^{\alpha} \sqrt{\Delta}\left(\frac{C}{A}\right)^{(z-1) /(2 \varepsilon)} \frac{1}{2 \pi \mathrm{i}} \int_{\mathrm{Br}^{\prime}} \frac{1}{\eta-B} \frac{1}{\sqrt{p(\eta)}} \exp \left(\frac{1}{\varepsilon}(z-1) g(\eta)\right) \mathrm{d} \eta,
$$

where

$$
g(\eta)=\frac{(\eta-B) \Omega}{2 \Delta}+\frac{1}{\eta-B}\left(p(\eta)+\eta \ln \left(\frac{\eta-p(\eta)}{\beta}\right)-\Delta-B \ln \left(\frac{B-\Delta}{\beta}\right)\right)
$$

and $\mathrm{Br}^{\prime}$ is a vertical contour in the complex plane with $\operatorname{Re}(\eta)>B$. For $\varepsilon \rightarrow 0$, with $\Omega$ fixed, we can evaluate (60) by the saddle point method to obtain

$$
\Phi_{l}(x) \sim \mu_{\infty} r^{\alpha} \sqrt{\Delta}\left(\frac{C}{A}\right)^{(z-1) / 2 \varepsilon} \sqrt{\frac{\varepsilon}{2 \pi(z-1)}} \frac{1}{\eta^{*}-B} \exp \left(\frac{1}{\varepsilon}(z-1) g\left(\eta^{*}\right)\right) \frac{1}{\sqrt{p\left(\eta^{*}\right) g^{\prime \prime}\left(\eta^{*}\right)}},
$$

where the saddle point $\eta^{*}(\Omega)$ is defined by $g^{\prime}\left(\eta^{*}\right)=0$. Note that $\eta^{*}(\Omega)>B$ for $\Omega<1$, i.e. the saddle point $\eta^{*}(\Omega)$ lies to the right of the pole at $\eta=B$ and the integrand is analytic for $\operatorname{Re}(\eta)>B$. Taking the derivative of $(61)$, we find that

$$
g^{\prime}(\eta)=\frac{\Omega}{2 \Delta}+\frac{1}{(\eta-B)^{2}}\left(\Delta+B \ln \left(\frac{B-\Delta}{\eta-p(\eta)}\right)-p(\eta)\right),
$$

and we observe that $g^{\prime}\left(\eta^{*}\right)=0$ if $\eta^{*}=B$ and $\Omega=1$, which implies that $\eta^{*}(1)=B$. To determine $\eta^{*}$ for $\Omega \sim 1$, we use (62) and an expansion of the form

$$
\eta^{*}(\Omega) \sim B+a_{1}(\Omega-1)+a_{2}(\Omega-1)^{2}+a_{3}(\Omega-1)^{3}+\cdots .
$$

Using (63) in (62) and expanding the latter in powers of $\Omega-1$, we find that

$$
a_{1}=-\frac{3 \Delta^{2}}{2 B}, \quad a_{2}=-\frac{27 \Delta^{2}}{32 B^{3}}\left(\Delta^{2}-3 B^{2}\right),
$$

and

$$
\begin{array}{rlrl}
g\left(\eta^{*}\right) & \sim \ln \left(\frac{B-\Delta}{\beta}\right)-\frac{3 \Delta}{8 B}(\Omega-1)^{2}, & & g^{\prime \prime}\left(\eta^{*}\right) \sim \frac{B}{3 \Delta^{3}}, \\
\frac{1}{\eta^{*}-B} \sim-\frac{2 B}{3 \Delta^{2}}(\Omega-1)^{-1}, & \frac{1}{\sqrt{p\left(\eta^{*}\right)}} \sim \frac{1}{\sqrt{\Delta}},
\end{array}
$$


from which we conclude that

$$
\Phi_{l}(x) \sim-\mu_{\infty} r \sqrt{\frac{2 B}{3 \pi \Delta(l-\alpha)}}(\Omega-1)^{-1} .
$$

Using (55) in (64), we obtain, for $x, l \rightarrow \infty$ with $\Omega \rightarrow 1$,

$$
F_{l}^{(1)}(x) \sim-(1-\rho) \exp \left(\frac{z \ln (\rho)}{\varepsilon}\right) \sqrt{\frac{2(\mu+\lambda) \varepsilon}{3 \pi(\mu-\lambda)(z-1)}}(\Omega-1)^{-1} .
$$

This must agree with the behavior of the ray expansion in $R^{\mathrm{C}}$ as $(y, z) \rightarrow(0,1)$. Next we evaluate $K$ and $\psi$ in (10) near the corner $(0,1)$. From (17) we have

$$
t \sim \frac{z-1}{\mu-\lambda}-\frac{(z-1)^{2}(\mu+\lambda)}{2(\mu-\lambda)^{3}} s, \quad s \rightarrow 0^{+} .
$$

Using (66) in (16), we obtain

$$
y \sim \frac{(z-1)^{2}}{2(\mu-\lambda)}-\frac{(z-1)^{3}(\mu+\lambda)}{3(\mu-\lambda)^{3}} s, \quad s \rightarrow 0^{+},
$$

or, using (58),

$$
s \sim-\frac{3}{2} \frac{(\mu-\lambda)^{2}}{(\mu+\lambda)} \frac{\Omega-1}{z-1}, \quad z \rightarrow 1 .
$$

We expand (20) for small $t$,

$$
\psi(s, t) \sim \ln (\rho)(1+(\mu-\lambda) t), \quad t \rightarrow 0,
$$

which taking (66) into account gives

$$
\Psi(y, z) \sim \ln (\rho)+(z-1) \ln (\rho), \quad z \rightarrow 1,
$$

in agreement with the exponential part of (65).

From (30) and (31), we obtain

$$
K(s, t) \sim K_{0}(s) \sqrt{\frac{3}{\left(\mu^{2}-\lambda^{2}\right) t^{3}}}, \quad t \rightarrow 0,
$$

or, using (66) and (67) in the above,

$$
\mathbb{K}(y, z) \sim K_{0}\left(-\frac{3}{2} \frac{(\mu-\lambda)^{2}}{(\mu+\lambda)} \frac{\Omega-1}{z-1}\right) \sqrt{\frac{3(\mu-\lambda)^{3}}{\left(\mu^{2}-\lambda^{2}\right)(z-1)^{3}}}, \quad z \rightarrow 1 .
$$

Matching the algebraic factors in (65) and (68) yields

$$
K_{0}(s)=\sqrt{\frac{\mu-\lambda}{2 \pi}} \frac{1-\rho}{s}
$$

and $v=\frac{1}{2}$. This completes the determination of the asymptotic solution corresponding to rays from the point $(0,1)$. To summarize, we have established the following result. 
Result 1. The solution of (8) is asymptotically given by

$$
\begin{gathered}
G(y, z) \sim \sqrt{\varepsilon} \exp \left(\frac{1}{\varepsilon} \Psi(y, z)\right) \mathbb{K}(y, z) \quad \text { in } R^{\mathrm{C}}, \\
G(\infty, z)-G(y, z) \sim-\sqrt{\varepsilon} \exp \left(\frac{1}{\varepsilon} \Psi(y, z)\right) \mathbb{K}(y, z) \quad \text { in } R,
\end{gathered}
$$

with

$$
\begin{gathered}
G(\infty, z)=(1-\rho) \exp \left(\frac{1}{\varepsilon} z \ln (\rho)\right) \\
R=\{0 \leq y, 0 \leq z \leq 1\} \cup\left\{Y_{0}(z) \leq y, 1 \leq z\right\}, \quad Y_{0}(z)=\frac{(z-1)^{2}}{2(\mu-\lambda)}, \quad z \geq 1, \\
\Psi(y, z)=\psi(s, t)=2 y s+(\ln (\rho)-s t)(z-1)+\ln (\rho), \\
\mathbb{K}(y, z)=K(s, t)=\sqrt{\frac{\mu-\lambda}{2 \pi J(s, t)}} \frac{1-\rho}{s},
\end{gathered}
$$

where $(y, z)$ is related to $(s, t)$ by $(16)$ and $(17)$, and $J(s, t)$ was defined in (26). We note that $s<0$ in $R$, so that the right-hand side of (70) is positive. This gives the leading term for the probability

$$
\operatorname{Pr}\left[X(\infty)>x=\frac{y}{\varepsilon}, Z(\infty)=k=\frac{z}{\varepsilon}\right]
$$

that the buffer exceeds $x=c y$. In the corner range where (36) applies, the leading term is given by (56) or (57).

\section{Transition layer}

We shall find a transition layer solution near the curve $y=Y_{0}(z)$, defined by (18), which separates $R$ and $R^{\mathrm{C}}$. On this curve $s=0$ and, hence, (72) is not valid because $\mathbb{K}(y, z)$ is infinite there.

We introduce the new function $L_{k}(x)$ defined by $F_{k}(x)=F_{k}(\infty) L_{k}(x)$. Then, for $L_{k}(x)$, (4) yields the equation

$$
(k-c) L_{k}^{\prime}=\mu L_{k-1}+\lambda L_{k+1}-(\lambda+\mu) L_{k},
$$

and boundary condition (7) becomes $L_{k}(\infty)=1$.

In terms of the variables $y=\varepsilon^{2} x$ and $z=\varepsilon k$, the function $L^{(1)}(y, z)=L_{k}(x)$ satisfies

$$
\varepsilon(z-1) \frac{\partial L^{(1)}}{\partial y}(y, z)=\mu L^{(1)}(y, z-\varepsilon)+\lambda L^{(1)}(y, z+\varepsilon)-(\lambda+\mu) L^{(1)}(y, z),
$$

and, therefore, as $\varepsilon \rightarrow 0$,

$$
(z-1) \frac{\partial L^{(1)}}{\partial y}=(\lambda-\mu) \frac{\partial L^{(1)}}{\partial z}+\frac{\lambda+\mu}{2} \frac{\partial^{2} L^{(1)}}{\partial z^{2}} \varepsilon+O\left(\frac{\partial^{3} L^{(1)}}{\partial z^{3}} \varepsilon^{2}\right) .
$$

Introducing the stretched variable $\varrho$, defined by

$$
y=Y_{0}(z)+\sqrt{\varepsilon} \varrho=\frac{(z-1)^{2}}{2(\mu-\lambda)}+\sqrt{\varepsilon} \varrho,
$$


and the function $L^{(2)}(\varrho, z)=L^{(1)}(y, z)$, we obtain, for $L^{(2)}(\varrho, z)$, to leading order, the diffusion equation

$$
(\lambda-\mu) \frac{\partial L^{(2)}}{\partial z}+\frac{(\lambda+\mu)}{(\lambda-\mu)^{2}}(z-1)^{2} \frac{\partial^{2} L^{(2)}}{\partial \varrho^{2}}=0 .
$$

To solve (73), we assume that $L^{(2)}(\varrho, z)$ is a function of the similarity variable $V=\varrho / r(z)$, and let $\mathfrak{L}(V)=L^{(2)}(\varrho, z)$, where $r(z)$ is not yet determined. From (73) we obtain

$$
-(\lambda-\mu) r(z) r^{\prime}(z) V \mathfrak{L}^{\prime}+\frac{(\lambda+\mu)}{(\lambda-\mu)^{2}}(z-1)^{2} \mathfrak{L}^{\prime \prime}=0,
$$

and $L_{k}(\infty)=1$ gives $\mathfrak{L}(\infty)=1$. We can eliminate $z$ in (74) by choosing $r(z)$ to satisfy the equation

$$
-(\lambda-\mu) r(z) r^{\prime}(z)=\frac{(\lambda+\mu)}{(\lambda-\mu)^{2}}(z-1)^{2} .
$$

We choose $r(1)=0$, which is necessary for matching the transition layer with the corner layer solution, (56), and solve (75) to obtain

$$
r(z)=\sqrt{\frac{2}{3} \frac{\mu+\lambda}{(\mu-\lambda)^{3}}}(z-1)^{3 / 2} .
$$

Now (74) becomes $\mathfrak{L}^{\prime \prime}=-V \mathfrak{L}^{\prime}$, with solution

$$
\mathfrak{L}(V)=\frac{1}{2}\left(1+\operatorname{erf}\left(\frac{V}{\sqrt{2}}\right)\right)=\frac{1}{\sqrt{2 \pi}} \int_{-\infty}^{V} \exp \left(-\frac{1}{2} u^{2}\right) \mathrm{d} u,
$$

since $\mathfrak{L}(\infty)=1$.

Thus, the transition layer solution for $y-Y_{0}(z)=O(\sqrt{\varepsilon})$ and $z>1$ is

$$
F_{k}(x) \sim(1-\rho) \rho^{k} \frac{1}{2}\left(1+\operatorname{erf}\left(\frac{V}{\sqrt{2}}\right)\right)
$$

with

$$
V(y, z)=\frac{y-Y_{0}(z)}{\sqrt{\varepsilon}} \sqrt{\frac{3}{2} \frac{(\mu-\lambda)^{3}}{\mu+\lambda}}(z-1)^{-3 / 2} .
$$

We can show that if (76) is expanded as

$$
V=\frac{y-Y_{0}(z)}{r(z) \sqrt{\varepsilon}} \rightarrow-\infty,
$$

the transition layer matches to the ray expansion in $R^{\mathrm{C}}$, as given by (69), corresponding to rays emanating from $(0,1)$.

\section{The boundary layer at $z=0$}

The ray expansion in (70) does not satisfy the boundary conditions in (5). Thus, we re-examine the problem on the scale $z=O(\varepsilon)(k=O(1))$, with $y>0$. We consider solutions of (4) which have the asymptotic form

$$
F_{k}(x)-F_{k}(\infty)=F_{k}^{(3)}(y)-F_{k}(\infty) \sim \varepsilon^{\nu_{3}} \exp \left(\frac{1}{\varepsilon} \Psi(y, 0)\right) K_{k}^{(3)}(y) .
$$


Using (77) in (4) and expanding in powers of $\varepsilon$ gives, to leading order,

$$
0=\lambda K_{k-1}^{(3)}+\mu K_{k+1}^{(3)}+\left(\Psi_{y}(y, 0)-(\lambda+\mu)\right) K_{k}^{(3)},
$$

or, using (14),

$$
0=\lambda K_{k-1}^{(3)}+\mu K_{k+1}^{(3)}+(S(y, 0)-(\lambda+\mu)) K_{k}^{(3)},
$$

and from (5) we obtain

$$
\rho K_{-1}^{(3)}=K_{0}^{(3)}
$$

From (17) we have, along $z=0$,

$$
\mu \xi(y)+\lambda \xi^{-1}(y)+S(y, 0)-(\lambda+\mu)=0,
$$

where

$$
\xi(y)=\exp (S(y, 0) T(y, 0)),
$$

and, therefore, we can rewrite (78) as

$$
\frac{\rho K_{k-1}^{(3)}+K_{k+1}^{(3)}}{K_{k}^{(3)}}=\rho \xi^{-1}(y)+\xi(y) .
$$

Since $S(y, 0)<0$ and $T(y, 0)>0$, we see that $0<\xi(y)<1$ for all $y$. Using (81) in (16), we have

$$
\mu \xi(y)-\lambda \xi^{-1}(y)+\lambda-\mu-(\lambda+\mu) \ln (\xi(y))=S^{2}(y, 0) y,
$$

which combined with (80) gives

$$
\left(1-\xi^{-1}\right) \rho-(1-\xi)-(\rho+1) \ln (\xi)=\mu\left(\left(1-\xi^{-1}\right) \rho+(1-\xi)\right)^{2} y .
$$

Solving (82) subject to (79), we obtain

$$
K_{k}^{(3)}(y)=\left(\frac{\xi(y)-1}{1-\rho \xi^{-1}(y)} \xi^{k}(y)+\rho^{k} \xi^{-k}(y)\right) \bar{K}(y),
$$

with $\bar{K}(y)$ to be determined, and, hence,

$$
F_{k}^{(3)}(y)-F_{k}(\infty) \sim \varepsilon^{\nu_{3}} \exp \left(\frac{1}{\varepsilon} \Psi(y, 0)\right)\left(\frac{\xi(y)-1}{1-\rho \xi^{-1}(y)} \xi^{k}(y)+\rho^{k} \xi^{-k}(y)\right) \bar{K}(y) .
$$

Setting $k=z / \varepsilon$ and $F_{k}^{(3)}(y)-F_{k}(\infty)=G^{(1)}(y, z)$ in (84) and letting $\varepsilon \rightarrow 0$, we obtain

$$
G^{(1)}(y, z) \sim \varepsilon^{\nu_{3}} \exp \left(\frac{1}{\varepsilon} \Psi(y, 0)+\frac{1}{\varepsilon} z \ln \left(\frac{\rho}{\xi(y)}\right)\right) \bar{K}(y)
$$

or, using (14),

$$
G^{(1)}(y, z) \sim \varepsilon^{\nu_{3}} \exp \left(\frac{1}{\varepsilon} \Psi(y, 0)+\frac{1}{\varepsilon} z \frac{\partial \Psi}{\partial z}(y, 0)\right) \bar{K}(y) .
$$

From (22), (26), (70), and (81), we have

$$
G(y, z)-G(\infty, z) \sim \sqrt{\varepsilon} \exp \left(\frac{1}{\varepsilon} \Psi(y, 0)+\frac{1}{\varepsilon} z \frac{\partial \Psi}{\partial z}(y, 0)\right) \sqrt{\frac{\mu-\lambda}{2 \pi J_{0}(y) S(y, 0)}}(1-\rho)
$$


as $z \rightarrow 0$, with

$$
\begin{gathered}
\Psi(y, 0)=2 y S(y, 0)+\ln (\xi(y))<0, \quad J_{0}(y)=2\left(\mu \xi(y)-\frac{\lambda}{\xi(y)}\right) y-1<0, \\
S(y, 0)=(\lambda+\mu)-\mu \xi(y)-\lambda \xi^{-1}(y)<0 .
\end{gathered}
$$

Matching (85) and (86), we conclude that

$$
\nu_{3}=\frac{1}{2}, \quad \bar{K}(y)=\sqrt{\frac{\mu-\lambda}{2 \pi J_{0}(y) S(y, 0)}}(1-\rho) .
$$

Therefore, for $k=O(1)$ and $y>0$, we have

$$
\begin{aligned}
F_{k}^{(3)}(y)-F_{k}(\infty) \sim & \sqrt{\varepsilon} \exp \left(\frac{1}{\varepsilon} \Psi(y, 0)\right)(1-\rho) \\
& \times\left(\frac{\xi(y)-1}{1-\rho \xi^{-1}(y)} \xi^{k}(y)+\rho^{k} \xi^{-k}(y)\right) \sqrt{\frac{\mu-\lambda}{2 \pi J_{0}(y) S(y, 0)}},
\end{aligned}
$$

where $\xi(y)$ is defined implicitly by (83).

\section{The boundary $x=0$}

For $x=0$ and $k \leq\lfloor c\rfloor$, the values of $F_{k}(0)$ can be computed from the ray expansion, since $F_{k}(0)-F_{k}(\infty) \sim \sqrt{\varepsilon} \mathbb{K}(0, z) \exp ((1 / \varepsilon) \Psi(0, z))$ is well defined. For $x=0$ and $k \geq\lfloor c\rfloor+1$, we have $F_{k}(0)=0$ by (6). We now examine how this boundary condition is satisfied by considering the scale $y=O(\varepsilon)$ and $z>1$. Note that this part of the boundary is in the region $R^{\mathrm{C}}$.

From (17) we have

$$
\mathrm{e}^{s t}=\frac{(z-1) s+\mu+\lambda+\sqrt{((z-1) s+\mu+\lambda)^{2}-4 \lambda \mu}}{2 \mu}, \quad z>1 .
$$

Using (87) in (16), we obtain

$$
S(y, z)=\frac{z-1}{y}+\frac{1}{z-1}\left((\mu+\lambda) \ln \left(\frac{\mu}{(z-1)^{2}} y\right)+2 \lambda\right)+O\left(y \ln ^{2} y\right),
$$

and using (88) in (87), we obtain

$$
T(y, z)=\frac{1}{z-1} \ln \left(\frac{(z-1)^{2}}{\mu y}\right) y+O\left(y^{2} \ln ^{2} y\right)
$$

for $y \rightarrow 0^{+}$and $z>1$.

Using (88) and (89) in (71) and (72), we find that

$$
\begin{aligned}
\Psi(y, z) \sim & \tilde{\Psi}(y, z) \\
= & \ln (\rho)+(z-1)\left(\ln \left(\frac{\lambda y}{(z-1)^{2}}\right)+2\right) \\
& +\frac{1}{z-1}\left((\lambda+\mu) \ln \left(\frac{\mu y}{(z-1)^{2}}\right)+\lambda-\mu\right) y, \quad y \rightarrow 0 .
\end{aligned}
$$


Hence, we shall consider asymptotic solutions of the form

$$
F_{k}(x) \sim \varepsilon^{\sigma} \exp \left(\frac{1}{\varepsilon} \tilde{\Psi}(\varepsilon x, \varepsilon k)\right) \tilde{K}(u, \varepsilon k),
$$

where $u=\varepsilon x, u=O(1)$, and $\sigma$ and $\tilde{K}(u, z)$ are to be determined. Using (91) in (8), we obtain, to leading order,

$$
(z-1) \frac{\partial \tilde{K}}{\partial z}+u \frac{\partial \tilde{K}}{\partial u}+\tilde{K}=0 .
$$

The most general solution to (92) is

$$
\tilde{K}(u, z)=\frac{1}{u} \tilde{k}(\Xi), \quad \Xi=\frac{z-1}{u} .
$$

Hence,

$$
F_{k}(x) \sim \tilde{G}(u, z)=\varepsilon^{\sigma} \exp \left(\frac{1}{\varepsilon} \tilde{\Psi}(\varepsilon u, z)\right) \frac{1}{u} \tilde{k}(\Xi) .
$$

To find $\tilde{k}(\Xi)$ and $\sigma$, we shall match (93) with the corner layer solution, (56).

Recalling that $l-\alpha=(z-1) / \varepsilon$ and using the asymptotic formula (51), we obtain, as $\varepsilon \rightarrow 0$ with $\theta$ fixed,

$$
\begin{aligned}
J_{(z-1) / \varepsilon+(\lambda+\mu) / \theta}\left(\frac{2 \sqrt{\mu \lambda}}{\theta}\right) \sim & \sqrt{\frac{\varepsilon}{2 \pi(z-1)}} \\
& \times \exp \left(\left(\frac{z-1}{\varepsilon}+\frac{\lambda+\mu}{\theta}\right) \ln \left(\frac{\sqrt{\mu \lambda} \mathrm{e} \varepsilon}{\theta(z-1)}\right)-\frac{(\lambda+\mu)}{\theta}\right) .
\end{aligned}
$$

Using (94) and writing (56) in terms of $u=\varepsilon x$ and $z=1+(l-\alpha) \varepsilon$, we have

$$
\begin{aligned}
F_{l}^{(1)}(x) \sim(1- & \rho) \sqrt{\frac{\mu-\lambda}{\mu+\lambda}} \exp \left(\left(\frac{z+1}{2 \varepsilon}-\frac{\alpha}{2}\right) \ln (\rho)\right) \sqrt{\frac{\varepsilon}{2 \pi(z-1)}} \\
\times \frac{1}{2 \pi \mathrm{i}} & \int_{\mathrm{Br}}\left(\frac{1}{\theta} \exp \left(\frac{u \theta}{\varepsilon}+\left(\frac{z-1}{\varepsilon}+\frac{\lambda+\mu}{\theta}\right) \ln \left(\frac{\sqrt{\mu \lambda} \mathrm{e} \varepsilon}{\theta(z-1)}\right)\right)\right. \\
& \left.\times \Gamma\left(\frac{\lambda+\mu}{\theta}+1-\alpha\right) \exp \left(\frac{\lambda-\mu}{\theta}-\left(\frac{\lambda+\mu}{\theta}-\alpha\right) \ln \left(\sqrt{\rho} \frac{\lambda+\mu}{\theta}\right)\right)\right) \mathrm{d} \theta .
\end{aligned}
$$

To evaluate (95) asymptotically as $\varepsilon \rightarrow 0$, we shall use the saddle point method. We find that the integrand has a saddle point at $\theta=\Xi$, so that

$$
\begin{aligned}
F_{l}^{(1)}(x) \sim & \varepsilon(1-\rho) \sqrt{\frac{1-\rho}{1+\rho}} \frac{1}{2 \pi u} \frac{1}{\Xi} \Gamma\left(\frac{\lambda+\mu}{\Xi}+1-\alpha\right) \\
& \times \exp \left(\frac{\ln (\rho)}{\varepsilon}+\frac{u \Xi}{\varepsilon} \ln \left(\frac{\lambda \mathrm{e}^{2} \varepsilon}{\Xi^{2} u}\right)+\alpha \ln \left(\frac{\lambda+\mu}{\Xi}\right)\right) \\
& \times \exp \left(\frac{\lambda+\mu}{\Xi} \ln \left(\frac{\varepsilon}{\Xi u(\rho+1)}\right)+\frac{2 \lambda}{\Xi}\right)
\end{aligned}
$$


Writing (93) in terms of $\Xi$ and matching with (96), we obtain $\sigma=1$ and

$$
\begin{aligned}
\tilde{G}(u, z)= & \varepsilon(1-\rho) \sqrt{\frac{1-\rho}{1+\rho}} \frac{1}{2 \pi} \frac{1}{z-1} \Gamma\left(\frac{(\lambda+\mu) u}{z-1}+1-\alpha\right) \\
& \times \exp \left(\frac{\ln (\rho)}{\varepsilon}+\frac{z-1}{\varepsilon} \ln \left(\frac{\lambda \mathrm{e}^{2} \varepsilon u}{(z-1)^{2}}\right)+\alpha \ln \left(\frac{(\lambda+\mu) u}{z-1}\right)\right) \\
& \times \exp \left(\frac{(\lambda+\mu) u}{z-1} \ln \left(\frac{\varepsilon}{(\rho+1)(z-1)}\right)+\frac{2 \lambda u}{z-1}\right) \text { for } z>1 .
\end{aligned}
$$

Note that from (97) we have $\tilde{G}(u, \varepsilon k)=O\left(u^{k-\lfloor c\rfloor}\right)$ as $u \rightarrow 0, k \geq\lfloor c\rfloor+1$.

\section{The marginal distribution}

We shall now find the equilibrium probability that the buffer content exceeds $x$,

$$
M(x)=\operatorname{Pr}[X(\infty)>x]=1-\sum_{k=0}^{\infty} F_{k}(x)
$$

for various ranges of $x$.

\subsection{Approximation for $x=O(1)$}

In this region we shall use the spectral representation of the corner layer solution. Using the generating function

$$
\sum_{i=-\infty}^{\infty} J_{i}(x) z^{i}=\exp \left(\frac{x}{2}\left(z-\frac{1}{z}\right)\right)
$$

in the form

$$
\exp \left(\frac{1-\rho}{\rho+1}(j+1-\alpha)\right)=(\sqrt{\rho})^{-(j+1)} \sum_{l=-\infty}^{\infty} J_{l-(j+1)}\left(-\frac{2 \sqrt{\rho}}{\rho+1}(j+1-\alpha)\right)(\sqrt{\rho})^{l},
$$

we obtain, from (57),

$$
\begin{aligned}
M(x) \sim & M^{(1)}(x) \\
= & (1-\rho) \rho^{c-\alpha+1} \sqrt{\frac{1-\rho}{1+\rho}} \\
& \times \sum_{j \geq 0} \frac{(j+1-\alpha)^{j}}{j !} \rho^{j} \exp \left(-\frac{x(\lambda+\mu)}{j+1-\alpha}+\frac{1-3 \rho}{\rho+1}(j+1-\alpha)\right) .
\end{aligned}
$$

\subsection{Approximation for $x=O\left(\varepsilon^{-2}\right)=O\left(c^{2}\right)$}

We shall now use the asymptotic solution in region $R$, as given by (70). We have

$$
M(x) \sim M^{(2)}(y)=-\sum_{k=0}^{\infty} G(y, k \varepsilon) \sim-\frac{1}{\sqrt{\varepsilon}} \int_{0}^{\infty} \exp \left(\frac{1}{\varepsilon} \Psi(y, z)\right) \mathbb{K}(y, z) \mathrm{d} z .
$$


To evaluate (99) as $\varepsilon \rightarrow 0$, we use the Laplace method. From (14) we obtain

$$
\Psi_{z}(y, z)=q=0 \Longleftrightarrow s t=\ln (\rho),
$$

and, therefore, the main contribution to (99) comes from $z=1$ and we obtain

$$
M^{(2)}(y) \sim-\frac{\sqrt{2 \pi}}{\sqrt{-\Psi_{z z}(y, 1)}} \exp \left(\frac{1}{\varepsilon} \Psi(y, 1)\right) \mathbb{K}(y, 1) .
$$

Using (87) in (26)-(27) and (71)-(72), we obtain

$$
\begin{gathered}
\Psi_{z z}(y, 1)=\frac{S(y, 1)}{\mu-\lambda}, \quad \mathbb{K}(y, 1)=\frac{1-\rho}{2 S(y, 1)} \sqrt{\frac{-S(y, 1)}{\pi y}}, \\
\Psi(y, 1)=2 y S(y, 1)+\ln (\rho),
\end{gathered}
$$

while (16) gives

$$
S(y, 1)=-\frac{\zeta}{\sqrt{y}}
$$

where

$$
\zeta=\sqrt{2(\lambda-\mu)-(\lambda+\mu) \ln (\rho)}
$$

Thus,

$$
M^{(2)}(y) \sim \sqrt{\frac{\mu-\lambda}{2}} \frac{1-\rho}{\zeta} \exp \left(\frac{1}{\varepsilon}(-2 \zeta \sqrt{y}+\ln \rho)\right) .
$$

\section{Summary and discussion}

In most of the domain $\mathfrak{D}=\{(y, z): y, z \geq 0\}$, the asymptotic expansion of $F_{k}(x)=G(y, z)$ is given by

$$
G(y, z) \sim \sqrt{\varepsilon} \exp \left(\frac{1}{\varepsilon} \Psi(y, z)\right) \mathbb{K}(y, z) \quad \text { in } R^{\mathrm{C}}
$$

or

$$
G(\infty, z)-G(y, z) \sim-\sqrt{\varepsilon} \exp \left(\frac{1}{\varepsilon} \Psi(y, z)\right) \mathbb{K}(y, z) \quad \text { in } R .
$$

If we consider the continuous part of the density, given by

$$
f_{k}(x)=F_{k}^{\prime}(x)=\varepsilon^{2} \frac{\partial G}{\partial y}(y, z), \quad x>0,
$$

the transition between $R$ and $R^{\mathrm{C}}$ disappears, and we have

$$
f_{k}(x) \sim \varepsilon^{3 / 2} \Psi_{y}(y, z) \exp \left(\frac{1}{\varepsilon} \Psi(y, z)\right) \mathbb{K}(y, z)=\varepsilon^{3 / 2} \exp \left(\frac{1}{\varepsilon} \psi(s, t)\right) s K(s, t),
$$

everywhere in the interior of $\mathfrak{D}$. Note that $\mathbb{K}(y, z)$ becomes infinite along $y=Y_{0}(z)$ (i.e. $s=0$ ), but the product $\Psi_{y}(y, z) \mathbb{K}(y, z)$ remains finite.

The asymptotic expansion of the boundary probabilities $F_{k}(0), k \leq\lfloor c\rfloor$, can be obtained by setting $y=0$ in (101). This expression can be used to estimate the difference

$$
F_{k}(\infty)-F_{k}(0)=\operatorname{Pr}\left[X(\infty)>0, Z(\infty)=k=\frac{z}{\varepsilon}\right]
$$




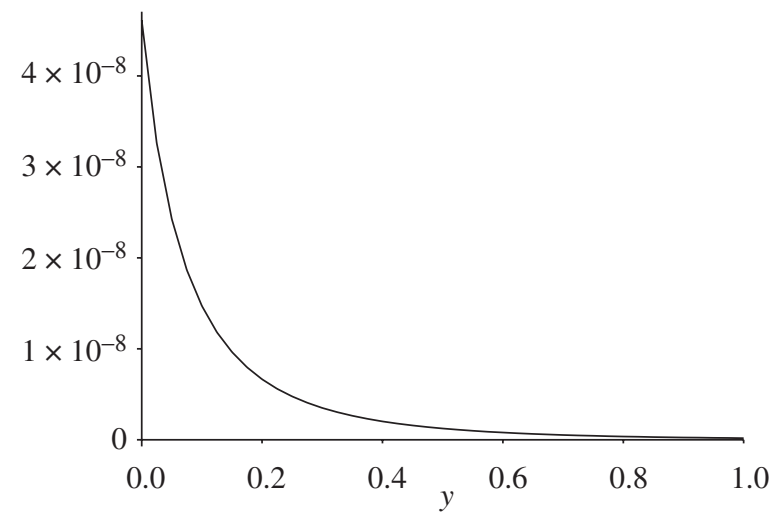

FIGURE 3: A plot of $\varepsilon^{3 / 2} \Psi_{y}(y, z) \exp ((1 / \varepsilon) \Psi(y, z)) \mathbb{K}(y, z)$ with $\varepsilon=0.1, \lambda=0.3145, \mu=0.8473$, and $z=0.5$.

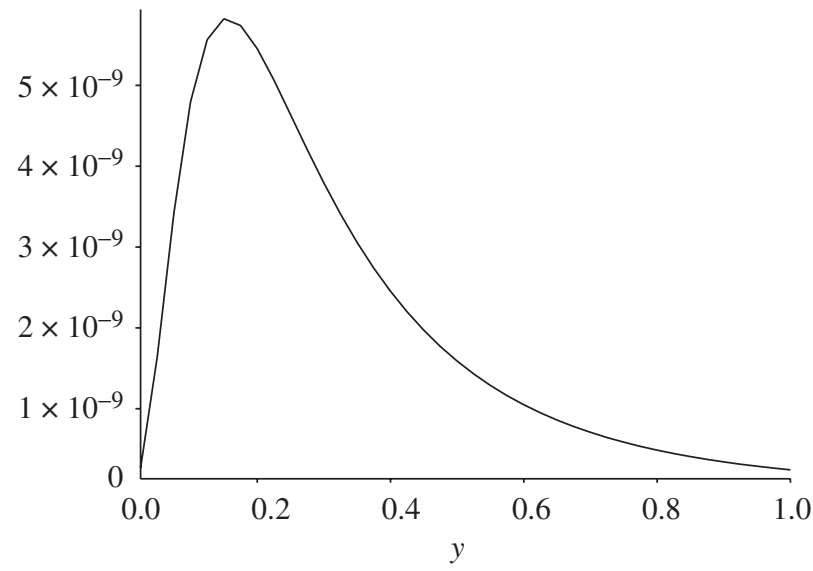

FIGURE 4: A plot of $\varepsilon^{3 / 2} \Psi_{y}(y, z) \exp ((1 / \varepsilon) \Psi(y, z)) \mathbb{K}(y, z)$ with $\varepsilon=0.1, \lambda=0.3145, \mu=0.8473$, and $z=1.5$.

which is exponentially small for $\varepsilon \rightarrow 0$. Also, for a fixed $z \in[0,1), f_{k}(x)$ is maximal at $x=0$ (see Figure 3). In other words, if $k<c$, the buffer will most likely be empty.

For a fixed $z>1, f_{k}(x)$ is peaked along the curve $y=Y_{0}(z)$ (see Figure 4). To see this better, we can use (19), (25), and (26) in (102), obtaining

$$
f_{k}(x) \sim \varepsilon^{3 / 2} \frac{1-\rho}{\sqrt{2 \pi}} \sqrt{\chi(z)} \exp \left(\frac{1}{\varepsilon}\left(z \ln (\rho)-\chi(z)\left(y-Y_{0}\right)^{2}\right)\right), \quad z>1
$$

with

$$
\chi(z)=\frac{3(\mu-\lambda)^{3}}{(\mu+\lambda)(z-1)^{3}}
$$




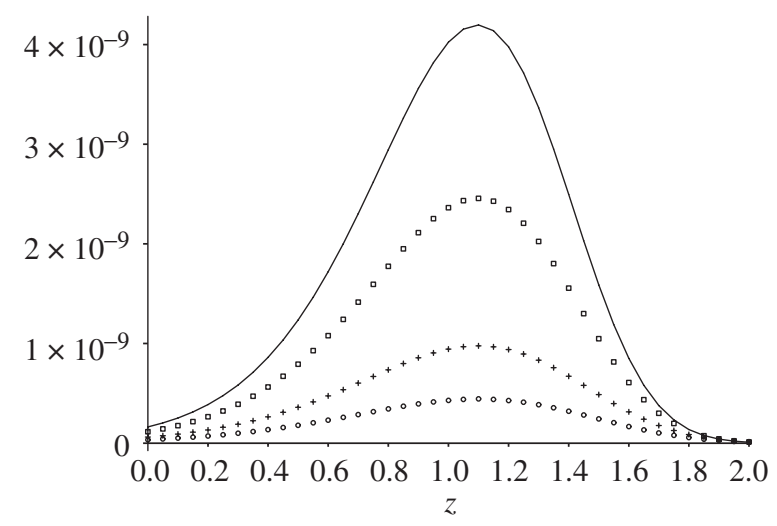

FIGURE 5: A plot of $\varepsilon^{3 / 2} \Psi_{y}(y, z) \exp ((1 / \varepsilon) \Psi(y, z)) \mathbb{K}(y, z)$ with $\varepsilon=0.1, \lambda=0.3145$, and $\mu=0.8473$ for $y=0.5$ (solid line), $y=0.6$ (squares), $y=0.8$ (crosses), and $y=1$ (circles).

or, equivalently,

$$
f_{k}(x) \sim(1-\rho) \rho^{k} \sqrt{\frac{\chi(k / c)}{2 \pi c^{3}}} \exp \left(-\frac{\chi(k / c)}{c^{3}}\left(x-c^{2} Y_{0}\left(\frac{k}{c}\right)\right)^{2}\right), \quad z>1 .
$$

This means that, given $k>c$ active sources, the most likely value of the buffer will be $x=$ $c^{2} Y_{0}(k / c)$.

For a fixed $x \geq 0, f_{k}(x)$ achieves its maximum around $z=1$ (see Figure 5). To find an expression for $f_{k}(x)$ when $z$ is close to 1 , we use (100) and obtain, for fixed $y>0$,

$$
f_{k}(x) \sim \varepsilon^{3 / 2} \frac{1-\rho}{2} \sqrt{\frac{\zeta}{\pi}} y^{-3 / 4} \exp \left(\frac{1}{\varepsilon}\left(\ln \rho-2 \zeta \sqrt{y}-\frac{\zeta(z-1)^{2}}{2 \sqrt{y}(\mu-\lambda)}\right)\right),
$$

or

$$
f_{k}(x) \sim \frac{1}{2}(1-\rho) \rho^{c} \sqrt{\frac{\zeta}{\pi}} x^{-3 / 4} \exp \left(-2 \zeta \sqrt{x}-\frac{\zeta(k-c)^{2}}{2 \sqrt{x}(\mu-\lambda)}\right) .
$$

\section{Acknowledgements}

This work was completed while D. Dominici was visiting Technische Universität Berlin and supported in part by a Sofja Kovalevskaja Award from the Humboldt Foundation, provided by Professor Olga Holtz. He wishes to thank Olga for her generous sponsorship and his colleagues at TU Berlin for their continuous help.

The work of C. Knessl was partly supported by NSF grant DMS 05-03745.

\section{References}

[1] Adan, I. And Resing, J. (1996). Simple analysis of a fluid queue driven by an M/M/1 queue. Queueing Systems Theory Appl. 22, 171-174.

[2] Anick, D., Mitra, D. And Sondhi, M. M. (1982). Stochastic theory of a data-handling system with multiple sources. Bell System Tech. J. 61, 1871-1894.

[3] Barbot, N. and Sericola, B. (2002). Stationary solution to the fluid queue fed by an M/M/1 queue. J. Appl. Prob. 39, 359-369. 
[4] Dominici, D. And Knessl, C. (2005). Geometrical optics approach to Markov-modulated fluid models. Stud. Appl. Math. 114, 45-93.

[5] Hashida, O. and Fujika, M. (1973). Queueing models for buffer memory in store-and-forward systems. In Proc. Seventh Internat. Teletraffic Congress (Stockholm, June 1973), Paper 323, 7 pp.

[6] Keller, J. B. (1978). Rays, waves and asymptotics. Bull. Amer. Math. Soc. 84, 727-750.

[7] Lenin, R. B. And Parthasarathy, P. R. (2000). A computational approach for fluid queues driven by truncated birth-death processes. Methodol. Comput. Appl. Prob. 2, 373-392.

[8] McDonald, D. And Qian, K. (1998). An approximation method for complete solutions of Markov-modulated fluid models. Queueing Systems Theory Appl. 30, 365-384.

[9] Miyazawa, M. (1994). Palm calculus for a process with a stationary random measure and its applications to fluid queues. Queueing Systems Theory Appl. 17, 183-211.

[10] Parthasarathy, P. R., Vijayashree, K. V. and Lenin, R. B. (2002). An M/M/1 driven fluid queue-continued fraction approach. Queueing Systems 42, 189-199.

[11] Ren, Q. AND Kobayashi, H. (1992). A mathematical theory for transient analysis of communications networks. IEICE Trans. Commun. 12, 1266-1276.

[12] Ren, Q. and Kobayashi, H. (1995). Transient solutions for the buffer behavior in statistical multiplexing. Performance Evaluation 23, 65-87.

[13] Sericola, B. (2001). A finite buffer fluid queue driven by a Markovian queue. Queueing Systems Theory Appl. 38, 213-220.

[14] Sericola, B., Parthasarathy, P. R. and Vijayashree, K. V. (2005). Exact transient solution of an M/M/1 driven fluid queue. Internat. J. Comput. Math. 82, 659-671.

[15] Tanaka, T., Hashida, O. and Takahashi Y. (1995). Transient analysis of fluid model for ATM statistical multiplexer. Performance Evaluation 23, 145-162.

[16] TuCKER, R. C. F. (1988). Accurate method for analysis of a packet-speech multiplexer with limited delay. IEEE Trans. Commun. 36, 479-483.

[17] Van Doorn, E. A. And Scheinhardt, W. R. W. (1997). A fluid queue driven by an infinite-state birthdeath process. In Teletraffic Contributions for the Information Age, eds V. Ramaswami and P. Wirth, Elsevier, Amsterdam, pp. 465-475.

[18] Virtamo, J. and Norros, I. (1994). Fluid queue driven by an M/M/1 queue. Queueing Systems Theory Appl. 16, 373-386.

[19] WiJngaARD, J. (1979). The effect of interstage buffer storage on the output of two unreliable production units in series with different production rates. AIIE Trans. 11, 42-47. 